\title{
Respiratory dysfunction as first presentation of myasthenia gravis misdiagnosed as COVID-19
}

\author{
Giuseppe Scopelliti ${ }^{1}$ (D) Maurizio Osio ${ }^{2}$ (D) Massimo Arquati ${ }^{3} \cdot$ Leonardo Pantoni $^{1,2}$ (D)
}

Received: 6 September 2020 / Accepted: 10 October 2020 / Published online: 17 October 2020

(C) Fondazione Società Italiana di Neurologia 2020

\begin{abstract}
Background The outbreak of the coronavirus disease 2019 (COVID-19) has had profound impact on health care not only for its direct effects, but also because it deeply influenced the whole clinical practice and diagnostic pathways, particularly in the acute setting. Case report We present the case of a patient with respiratory dysfunction due to myasthenia gravis (MG) initially misdiagnosed as severe acute respiratory syndrome coronavirus 2 (SARS-CoV-2) infection due to ambiguity in the interpretation of radiological and microbiological findings during COVID-19 pandemic.

Discussion Respiratory dysfunction as first clinical manifestation of myasthenia gravis is rare, but potentially very harmful. Emergency physicians should always consider neurological diseases when dyspnea cannot be explained by cardiac or respiratory causes.
\end{abstract}

Keywords COVID-19 $\cdot$ SARS-CoV-2 $\cdot$ Myasthenia gravis $\cdot$ Dyspnea $\cdot$ Emergency medicine

\section{Introduction}

The coronavirus disease 2019 (COVID-19) pandemic caused by the severe acute respiratory syndrome coronavirus 2 (SARS$\mathrm{CoV}-2$ ) is a sanitary emergency that has required massive public health efforts and introduced new diagnostic and therapeutic challenges in the everyday practice. Here, we present the case of a patient with fluctuant dyspnea as first presentation of seronegative myasthenia gravis (MG) that was initially misdiagnosed as COVID-19 for his respiratory disturbances.

\section{Case report}

A 46-year-old man, with hypertension and past history of cigarette smoking, presented to the emergency department (ED) on March 26 for symptoms consistent with upper respiratory infection (cough, sore throat, and fever). The patient had been put on

Leonardo Pantoni

leonardo.pantoni@unimi.it

1 "Luigi Sacco" Department of Biomedical and Clinical Sciences, University of Milan, Via Giovanni Battista Grassi 74, 20157 Milan, Italy

2 Neurology Unit, Ospedale Luigi Sacco, Milan, Italy

3 Emergency Medicine, Ospedale Luigi Sacco, Milan, Italy azithromycin by the general practitioner 3 days before the presentation in the ED. Polymerase chain reaction (PCR) assay on nasal-pharyngeal swab sample was negative for SARS-CoV-2, while a chest X-ray was reported as suggestive for mild interstitial pneumonia. Thus, the patient was hospitalized in a COVID19 ward and eventually discharged 3 days after, afebrile and without signs of respiratory dysfunction. The diagnosis at discharge was "mild SARS-CoV-2-related pneumonia."

A few days after discharge, the patient suffered from shortness of breath, generalized weakness, and marked effort intolerance. Moreover, he occasionally found low values of arterial oxygen saturation on self-measurement at home (O2 saturation swinging from $91-97 \%$ over the day). For this reason, he presented to ED again on April 4, when another nasal-pharyngeal swab PCR for SARS-CoV-2 was negative. The chest CT scan showed modest bibasilar infiltrates (Fig. 1), and the arterial blood gases were within normal values. The diagnosis of SARS-CoV-2-related infection was confirmed, and the patient was discharged a few hours later in good general conditions.

On April 24, the patient returned to the ED for the third time complaining of persistence of asthenia and blood oxygen desaturation. He was hospitalized again and underwent another panel of investigations, including a PCR assay and a rapid Ig test for SARS-CoV-2 (both negative), an arterial gas analysis (SO2 97\%; pO2 $74 \mathrm{mmHg}$ [9,8 kPa]; pCO2 $44 \mathrm{mmHg}$ [5,8 kPa]; lactate $1.6 \mathrm{mmol} / \mathrm{L} ; \mathrm{HCO} 3-26 \mathrm{mmol} / \mathrm{L}$ ), an echocardiography (normal), a new chest CT scan (mild basilar 
Fig. 1 Chest CT scan. Bilateral lung infiltrates likely depicting atelectasis
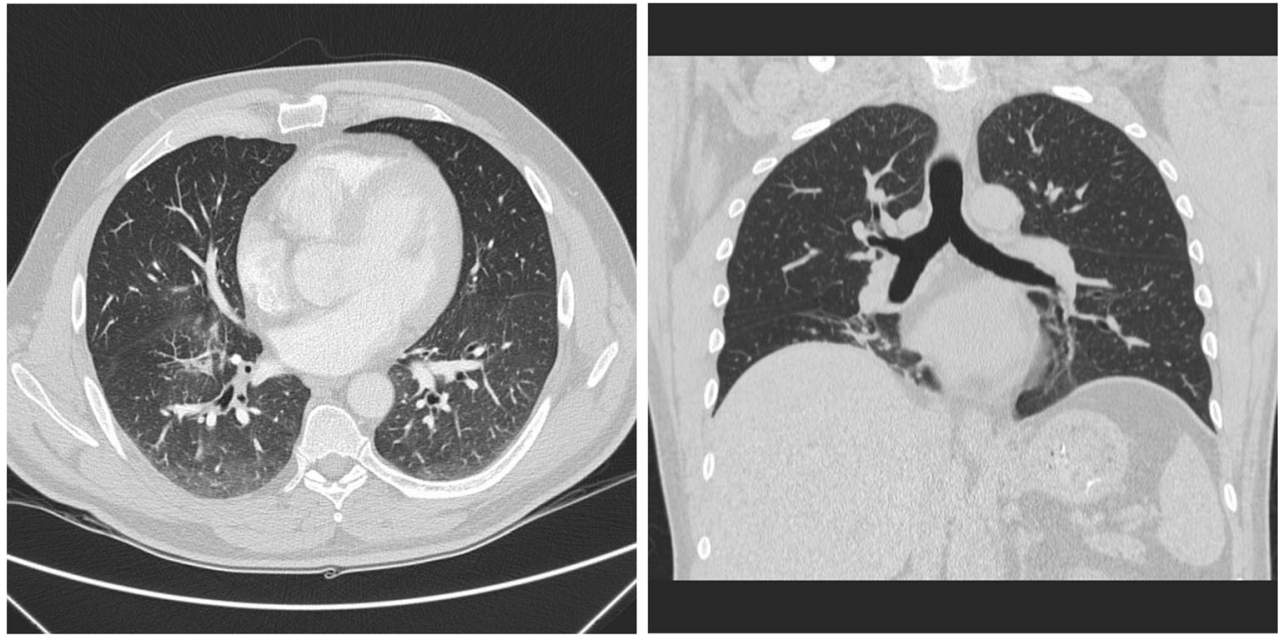

infiltrates), and a six-minute walking test (showing arterial oxygen desaturation down to $84 \%$ ).

Because the prevailing clinical features were fatigue and dyspnea during effort, with only mild pathological findings on chest CT scan, a neurological evaluation was requested at this time to rule out a neuromuscular condition. The consultant neurologist outlined slight hypophonia after sustained speech, slight diplopia, and ptosis in one eye during sustained upward gaze and slight weakness in proximal lower limb muscles (scored 4/5 in the Medical Research Council scale), with normal deep tendon reflexes. Given the presence of mild signs of neuromuscular fatigability, repetitive nerve stimulation was performed, showing $15 \%$ decrement in the amplitude of compound muscle action potential registered in right nasalis muscle while stimulating at low frequency $(5 \mathrm{~Hz})$ the facial nerve (Fig. 2), strongly supporting a diagnosis of generalized myasthenia gravis (MG).

Quickly after the introduction of anticholinesterase therapy and oral steroid, dyspnea and fatigue significantly improved. Moreover, the 6-minute walking test did not show arterial blood desaturation. Serological testing for antibodies against acetylcholine receptor (AChR), muscle-specific kinase (MUSK), and ryanodin receptor (RyR) was negative. The chest CT scan previously performed was reviewed and no thymic residual tissue was identified. The patient was discharged in good medical condition and put on pyridostigmine $240 \mathrm{mg}$ daily and oral prednisone $50 \mathrm{mg}$ daily. He was instructed to promptly recognize symptoms of muscular fatigability and respiratory failure. At 1-month followup, the patient was minimally symptomatic, complaining only mild effort intolerance; he was put on azathioprine, and tapering of oral steroid was planned after 1 month.

\section{Discussion}

Respiratory dysfunction as the first clinical manifestation of MG has been rarely described, although no ad
Fig. 2 Repetitive nerve stimulation. Facial nerve stimulation at $5 \mathrm{~Hz}$ showing a decrement of $15 \%$ in the amplitude of compound muscle action potential registered in right nasalis muscle

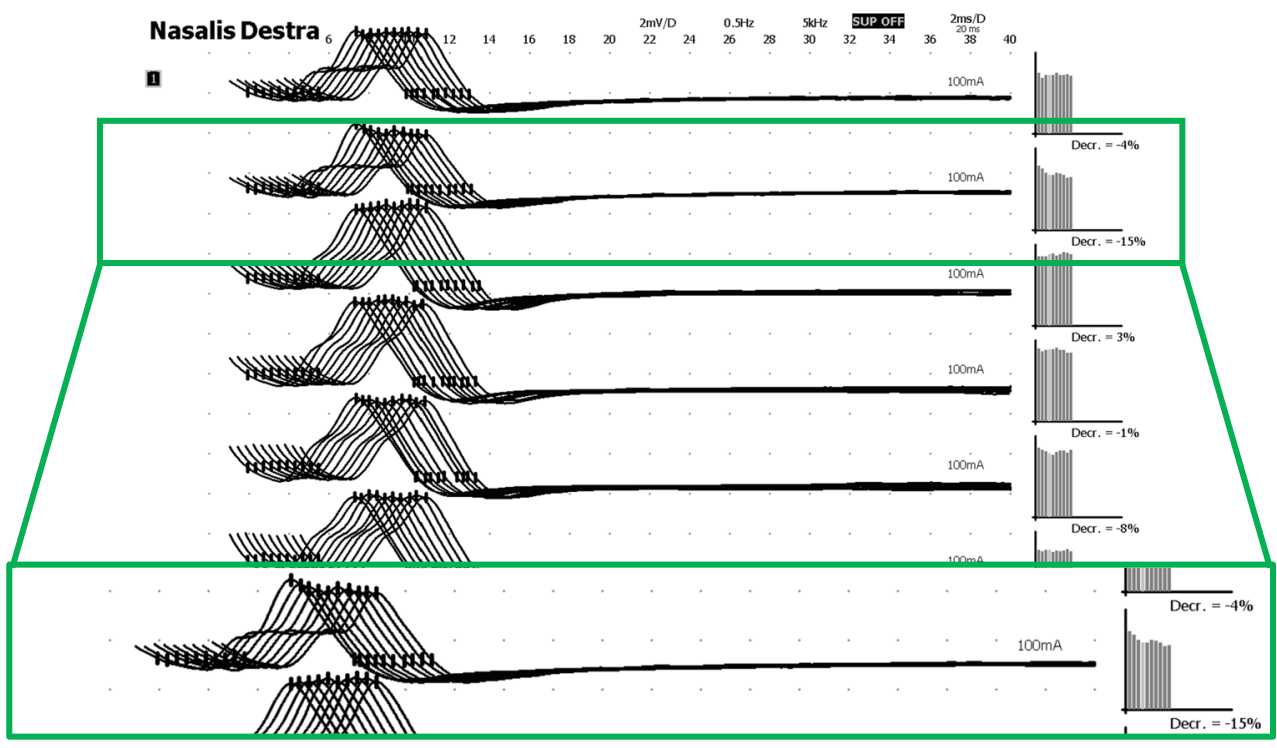


hoc study has estimated accurately the frequency of such clinical presentation [1].

Albeit respiratory failure in the context of MG can be extremely severe, in our case dyspnea was predominantly present during effort, and hypoxemia was never harmful. In our patient, MG was probably latent or paucisymptomatic (the patient previously complained of diplopia only if explicitly asked about it). Based on the presence of the symptoms at the very beginning of the clinical presentation including fever, we can hypothesize that the patient suffered an upper respiratory tract infection (not SARS-CoV-2-related) and shortly after developed respiratory muscles weakness due to MG. Azithromycin, which the patient took for 9 days when he had cough and fever, could have had a role in unmasking MG. Of note, hydroxychloroquine, which was widely used during the COVID-19 pandemic and is known to be associated with MG worsening [2], was not prescribed in this patient. Since we did not performed laryngoscopy to evaluate the function of vocal cords, we cannot rule out the occurrence of vocal cord paresis that may have both caused hypophonia and contributed to dyspnea in our patient. Ambiguity in the interpretation of radiological imaging (i.e., chest X-ray and chest CT scan) and microbiological testing (i.e., PCR assay and Ig rapid test), along with the due cautious approach to a case of acute dyspnea during the dramatic COVID-19 outbreak epidemic in an area severely hit by the disease, led to a delay in diagnosis. As it is well known, sensitivity of nasal swab PCR assay is moderate (estimated around 63-78\%) [3]; thus SARSCoV-2 infection could not be ruled out after multiple negative assays. The chest $\mathrm{CT}$ scan findings, however, were not specific for COVID-19; in fact, the bibasilar lung infiltrates likely depict atelectasis due to decreased respiratory muscles movement, hence representing a potential "radiological clue" of neuromuscular involvement [4]. In our case, antibodies against AChR and MUSK were tested negative: this condition, namely double-seronegative MG, occurs in about $15 \%$ of generalized MG and may be caused by rarer autoantibodies like anti-low-density lipoprotein receptor-related protein 4 (LRP4), and anti-cortactin. [5] Our case underlines the importance of considering neurological causes when dyspnea cannot be explained by cardiac or respiratory causes in the acute setting. Prompt recognition of MG in the case of acute respiratory dysfunction is crucial, and emergency physicians should always include MG in the differential diagnosis. Remarkably, looking for mild symptoms of neuromuscular fatigability such as fluctuant diplopia and ocular ptosis may improve diagnostic accuracy and help recognize generalized MG.

Authors' contribution All authors contributed equally to the paper.

\section{Compliance with ethical standards}

Conflict of interest The authors have no relevant financial or nonfinancial interests to disclose

Ethical Approval None

Informed consent The participant has consented to the submission of the case report to the journal.

\section{References}

1. Kim WH, Kim JH, Kim EK, Yun SP, Kim KK, Kim WC, Jeong HC (2010) Myasthenia gravis presenting as isolated respiratory failure: a case report. Korean J Intern Med. 25(1):101-104. https://doi.org/10. 3904/kjim.2010.25.1.101

2. Bird SJ (2020) Overview of the treatment of myasthenia gravis. In: Post TW (ed) UpToDate. UpToDate Inc, Waltham https://www. uptodate.com (Accessed on 20 May 2020)

3. Zitek T (2020) The appropriate use of testing for COVID-19. West J Emerg Med 21(3):470-472. Published 2020 Apr 13. https://doi.org/ 10.5811/westjem.2020.4.47370

4. Mandaliya R, Kulandaivel K, Nowotarski N et al (2015) A challenging diagnosis of fluctuating dyspnea: myasthenia gravis. J Clin Diagn Res 9(6):OD06-OOD8. https://doi.org/10.7860/JCDR/2015/ 12389.6015

5. Cortés-Vicente E, Gallardo E, Martínez MÁ, Díaz-Manera J, Querol L, Rojas-García R, Illa I (2016) clinical characteristics of patients with double-seronegative myasthenia gravis and antibodies to cortactin. JAMA Neurol 73(9):1099-1104. https://doi.org/10.1001/ jamaneurol.2016.2032

Publisher's note Springer Nature remains neutral with regard to jurisdictional claims in published maps and institutional affiliations. 